\title{
Energy Efficient Cross-Layer Transmission Model for Mobile Wireless Sensor Networks
}

\author{
Xin Yang, Ling Wang, and Jian Xie \\ Electronics and Information School, Northwestern Polytechnical University, Xian, China \\ Correspondence should be addressed to Xin Yang; x_yang119@126.com
}

Received 17 October 2016; Revised 26 February 2017; Accepted 7 March 2017; Published 19 March 2017

Academic Editor: Jinglan Zhang

Copyright (C) 2017 Xin Yang et al. This is an open access article distributed under the Creative Commons Attribution License, which permits unrestricted use, distribution, and reproduction in any medium, provided the original work is properly cited.

The performance of mobile wireless sensor networks (MWSN) witnessed a significant improvement in recent years, such as throughput, transmission delay, and collision detection. However, MWSN still suffers from high energy consumption, since most of the sensors or users in MWSN are based on passive devices. In order to remedy this problem, in this paper we present a Cross-layer Energy Efficiency (CEE) model for MWSN. CEE is a cross-layer model which contains three layers. It utilizes nodes location information in network layer, medium access control (MAC) protocol in MAC layer (sublayer of data link layer), and fullduplex interfaces in physical (PHY) layer. The CEE model offers a number of advantages in regard to energy efficiency, throughput improvement, low delay, and power control compared to existing models. According to the performance evaluation, the proposed transmission model effectively reduces energy consumption and improves other transmission performances. Also, it has been proved that the proposed model can be used in practical MWSN as Internet of things (IoT).

\section{Introduction}

Efforts have been made in the field of mobile wireless sensor networks (MWSN), which make contributions to the transmission performance, such as throughput, end-to-end delay, and packets delivery rate. However, in the case of not weakening other transmission performances, the MWSN still suffers from energy efficiency [1]. Many researchers focus on investigating the PHY layer to improve the performance. However, improvements are needed with the combination of PHY layer and higher layers. In other words, cross-layer transmission model is required.

Full-duplex interfaces are widely used in wireless sensor networks, since they have obvious advantages on the transmission performance [2]. Many researchers implement fullduplex devices on PHY layer. The consideration of this paper is that the performance on energy efficiency can be promoted by combining full-duplex interface techniques in PHY layer with MAC layer and network layer.

To improve the transmission performance of wireless sensor networks, a large number of models and protocols have been proposed. Service-availability-awareness is a cross-layer protocol based on sleep scheduling proposed for wireless sensor networks [3]. It has a significant contribution in prolonging the network lifetime. However, the track on duty-cycles of nodes increases energy consumption, and it sacrifices the performance of delay. For the MWSN, the protocol termed METC-ACW utilizes routing path to increase successful delivery ratio and prolong networks lifetime [4], but the application of the proposed cross-layer mechanism is limited. Location aware and fault tolerant clustering routing protocol is proposed based on location enhanced routing. It enhances the clustering mechanism and decreases the energy consumption but limits the networks' flexibility [5]. The model proposed in [6] is based on cross-layer networks and has a significant improvement on energy efficiency. Nevertheless, it suffers from heavy overhead. The protocol named STAIRS is proposed to solve transmission collision in single hop wireless sensor networks. Yet it suffers from collision in multihop networks [7]. An energy efficiency and quality of service (QoS) aware multipath routing model (EQSR) is proposed in [8] for MWSN. It is a cross-layer model based on routing path selection and PHY layer elements. However, the throughput and end-to-end delay need to be improved. 
In this paper, an energy efficient cross-layer transmission model for the MWSN is proposed. We concentrate on decreasing energy consumption of the networks, but we do not neglect the negative influence on other transmission performances brought by the reduction of energy consumption. On the contrary, the proposed transmission model not only reduces the energy consumption but also improves other transmission performances of the MWSN. The rest of the paper is organized as follows. The system model is provided in the second section; it includes introduction of nodes' interfaces and transmission scheme. In the third section, we present the transmission details of the MWSN. The analysis and simulation results are presented in the fourth section to demonstrate the advantages of the proposed model. Finally, the fifth section concludes this paper.

\section{System Model}

In our transmission model for MWSN, we assume that all nodes in the system are in Poisson distribution. There are two types of full-duplex transmission route in the proposed model. One is the bidirectional route, which means that node A transmits information to node $\mathrm{B}$ and node $\mathrm{B}$ transmits information to node $\mathrm{A}$ at the same time. The other is the unidirectional route, where node $\mathrm{A}$ transmits information to node $\mathrm{B}$ and node $\mathrm{B}$ transmits information to node $\mathrm{C}$. Each node in the model has two kinds of transmission range as shown in Figure 1. One is Data Transmission Range (DTR). The other is Sensing Transmission Range (STR). In a node's DTR, it can successfully send data to destination node. In a node's STR, it can sense the transmission of all sending nodes in the area. In this paper, we assume that both the STRs and the DTRs of the nodes are equal. The definition of different ranges can solve hidden nodes problem. As shown in Figure 1, node $\mathrm{F}$ is in the STR of node $\mathrm{C}$ but not of node $\mathrm{A}$, which may act as a hidden node in the transmission between nodes $\mathrm{A}$ and $\mathrm{C}$. Hence, in bidirectional route transmission, hidden nodes problem can be solved by full-duplex interface due to simultaneous transmission. In unidirectional route transmission, node $\mathrm{F}$ can sense the ongoing transmission from node C by RTS/CTS mechanism [9]. Hence, the problem is solved [10].

2.1. Interfaces of Nodes. In the MWSN, each node is equipped with two full-duplex interfaces, called S hopping interface and $\mathrm{R}$ hopping interface. Both of the interfaces can switch channels in multiple channel systems independently. The two interfaces of one node are in different hopping sequences and different channels. Hence, a node with two interfaces can simultaneously transmit and receive data packets without interfering with each other. In general, the S hopping interface is used to send messages, while the $\mathrm{R}$ hopping interface is for reception. In order to solve the busy receiver problem, we assumed that the $\mathrm{R}$ hopping interface stays in its hopping sequence and never deviates from it. In the proposed protocol, the R and S hopping sequence information of sender node and receiver node are contained in CTS and RTS in the transmission $[11,12]$. If the sender node wants to send message to receiver node, the $\mathrm{S}$ hopping node of sender node

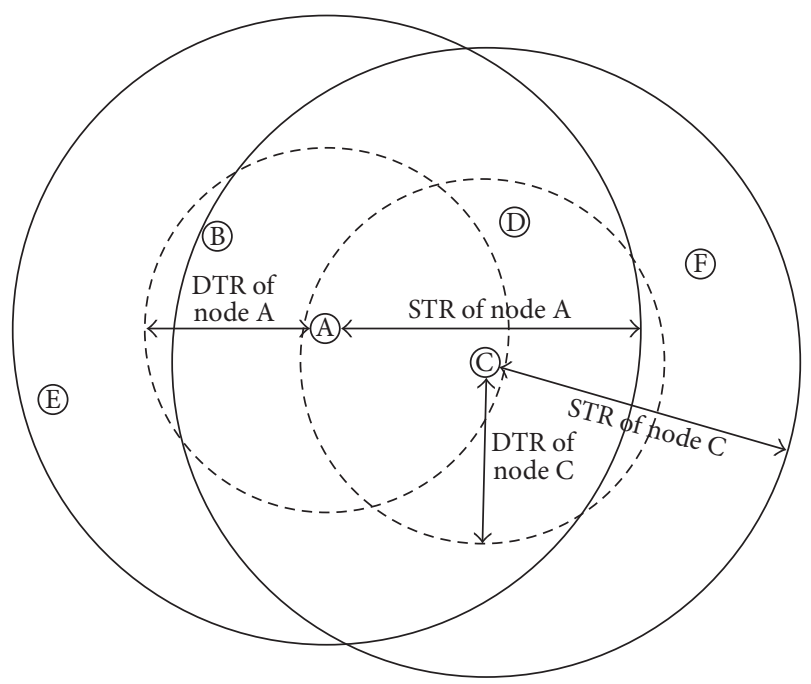

FIGURE 1: Definition of DTR and STR.

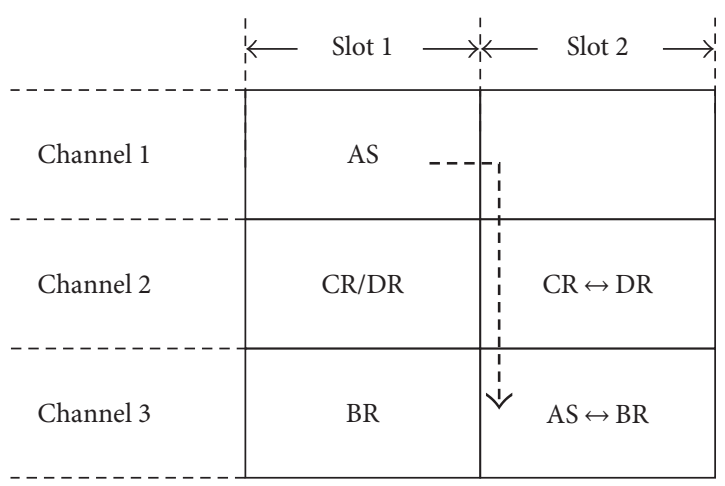

FIGURE 2: Transmission examples of interfaces.

switches to the $\mathrm{R}$ hopping node's channel of receiver node to complete the transmission. In particular, if the $\mathrm{R}$ hopping interfaces of both sender nodes and receiver nodes share the same channel, the sender node starts the transmission using its R hopping interface [13]. Therefore, they can transmit the packet directly without using $\mathrm{S}$ hopping interface of sender node.

As shown in Figure 2, BR, CR, and DR are the $\mathrm{R}$ hopping interfaces of nodes $\mathrm{B}, \mathrm{C}$, and $\mathrm{D}$, respectively, and AS represents the $S$ hopping interface of node $A$. If node $A$ wants to send messages to node B, the interface AS switches from channel 1 to channel 3 to meet BR. Then the transmission is completed in slot 2. Meanwhile, if node $C$ wants to send massages to node $\mathrm{D}$, because the interfaces $\mathrm{CR}$ and $\mathrm{DR}$ are both in channel 2 , the transmission can be completed directly.

2.2. Transmission Scheme. In this section, we will take one hop from sender node to destination node in unidirectional channel to illustrate the basic transmission scheme. The bidirectional route transmission and multihop unidirectional route transmission are all based on the basic transmission and will be described in the next section. The illustration of a cross-layer transmission for energy efficiency is shown in 


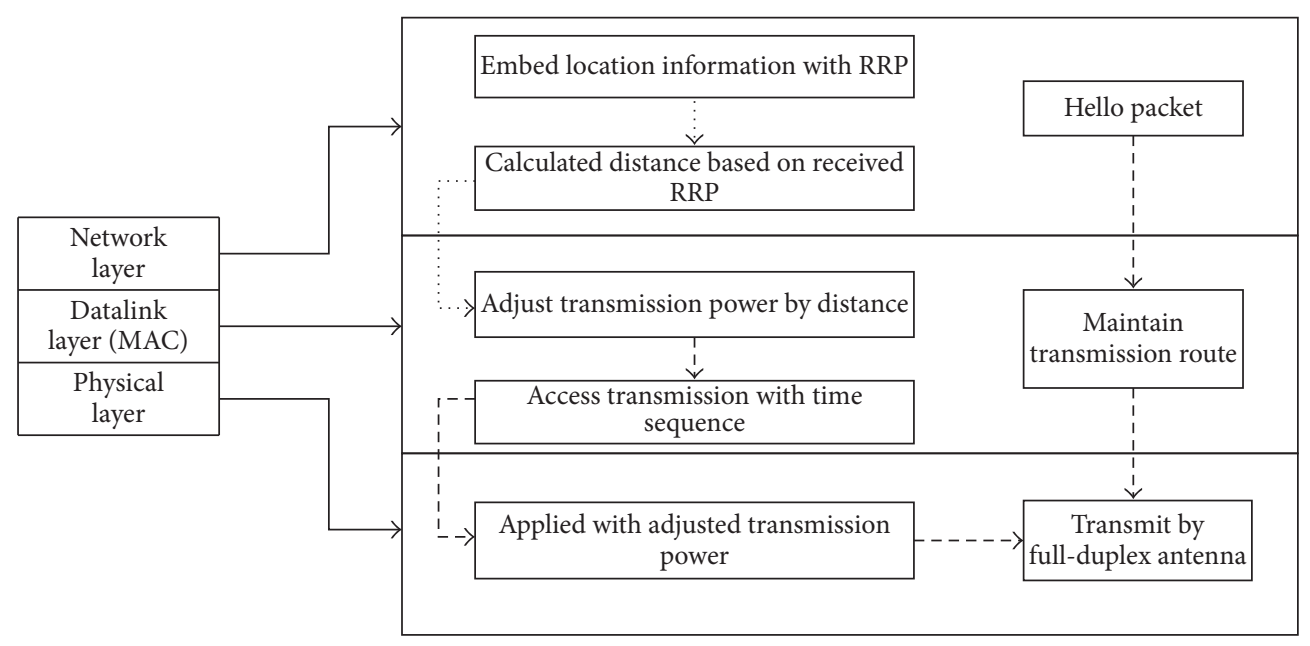

FIgURE 3: Cross-layer operational model.

Figure 3. Network layer, data link layer, and physical layer are involved in the operational model. The MAC layer is included in data link layer $[14,15]$. The practical deployment of crosslayer scheme in the proposed transmission model of sensor networks is an important task. We should not only design MAC layer protocol with network layer location information but also take PHY layer conditions into consideration, for example, the diversity reception of path loss transmission and the antijamming for transmission security and fullduplex technique in PHY layer. The appropriate PHY layer propagation models for different transmission environments are necessary to the practical deployment of sensor networks. The PHY layer propagation models are also needed to support upper layers to complete the whole transmission.

Firstly, at the initialization stage of the network, a sender node broadcast a discovery message to collect and store neighbor nodes' information. If the destination node is in sender node's DTR, the sender node sends a request-tosend (RTS) to the destination node to start the transmission [13]. Secondly, after receiving the RTS from sender node, the destination node waits for short interframe space (SIFS) duration and sends clear-to-send (CTS) to the sender node, attached with route request packets (RRP). The location information is embedded in RRP.

The purpose of RRP is to establish a stable route between the sender node and the destination node. The location information is provided by either GPS or any other position localization methods [16]. Hello packet is a periodic neighbor maintenance message which is utilized in the proposed model. Because hello packets are broadcast packets, it is possible to use neighbor nodes' information from the network layer in the data link layer. In RRP, a 32-bit field is required for the location information according to Virtual Earth's tiling system method [17], in which 16 bits are assigned to $x$-axis and 16 bits to $y$-axis. In addition, the structure of RRP is shown in Figure 4. Depending on the location information of nodes, sender node can calculate transmission power and range according to the Euclidean distance between sender and destination node. In order to reduce the energy used in

\begin{tabular}{|c|}
\hline Prefix (type, reserved, hop count) \\
\hline Destination IP address \\
\hline Destination sequence number \\
\hline Source IP address \\
\hline Lifetime \\
\hline Location information $(x$-axis and $y$-axis $)$ \\
\hline
\end{tabular}

FIGURE 4: RRP structure.

control packets and overhead, hello packets are only allowed to be broadcast in active routes, which are established to transmit data between sender and destination nodes. Finally, after the data transmission, destination node sends ACK to sender node [18].

Taking realistic models into consideration, the coverage of nodes and networks may be influenced. Just like the RTS/CTS in IEEE 802.11 MAC protocol, the proposed transmission model is also influenced by transmission path loss. In the practical deployment of sensor networks (e.g., the IoT), wireless transmission path loss transmission models are needed depending on different transmission conditions. For example, many IoT systems are equipped in cities, so Okumura propagation model [19] is considered in the deployment of the proposed protocol for cities. When the IoT systems are equipped indoors, the attenuation factor model can be used to solve the problem. Depending on the models we can compute the energy consumption, transmission range, and other parameters for the practical deployment of the proposed protocol.

\section{Transmission Details}

In full-duplex bidirectional route transmission, a sender node broadcasts discovery message, RTS message, and CTS message in its maximum power $P_{\max }$ in order to reach its STR and capture channel for transmission. It can also avoid 


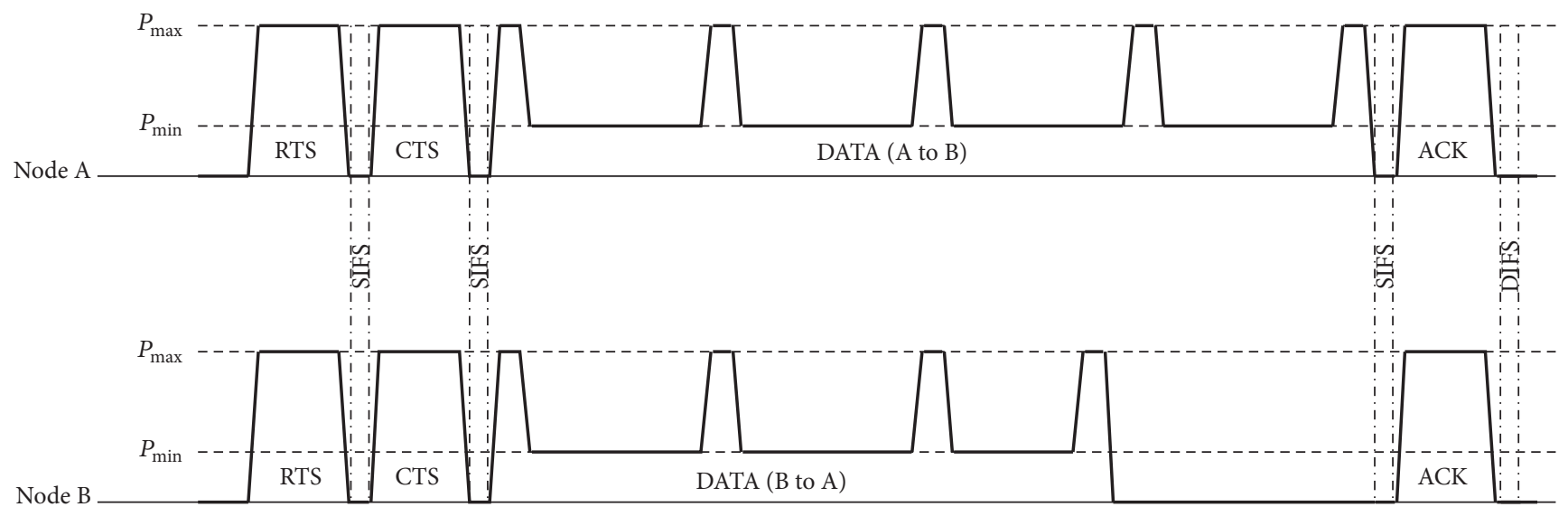

FIgURE 5: Bidirectional route transmission.

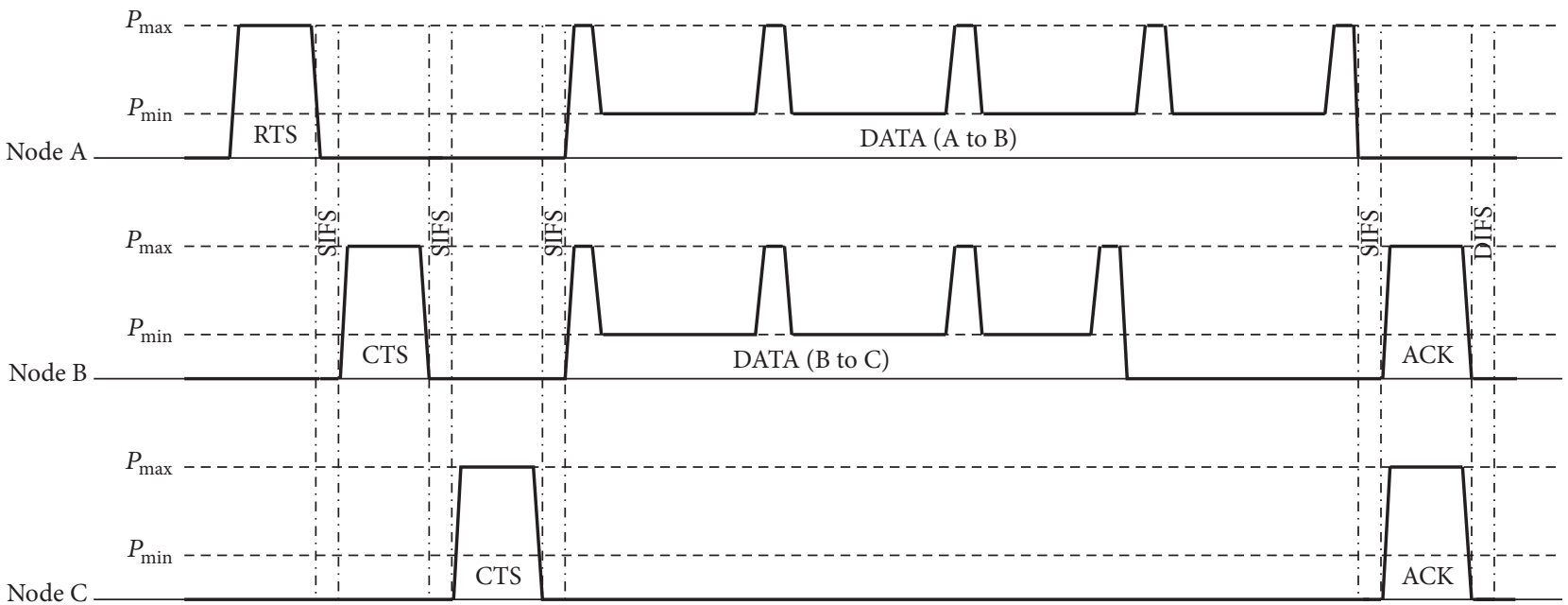

FIGURE 6: Unidirectional route transmission.

hidden terminal problem. When a sender node calculates the Euclidean distance of transmission, it transmits data in its $P_{\min }$ which is defined as the minimum power for successful data transmission. The relationship between $P_{\max }$ and $P_{\min }$ is shown as

$$
P_{\text {min }}=\frac{P_{\max }}{P_{\text {rec }}} \times R_{\text {min }}^{\mathrm{rec}} \times c,
$$

where $P_{\text {rec }}$ is the received power, $R_{\mathrm{min}}^{\mathrm{rec}}$ is the minimum necessary received signal strength, and $c$ is a constant similar in [20], and we set $c=1$ in our simulation and analysis.

The examples of bidirectional route and unidirectional route transmission scheme in MAC layer of the proposed model are shown in Figures 5 and 6, respectively.

In bidirectional route transmission (as shown in Figure 5), the full-duplex nodes A and B send RTS synchronically after initialization. After SIFS, they send CTS attached with their location information which is obtained from network layer. Both RTS and CTS are transmitted in the maximum power. After another SIFS, data is transmitted in their minimum power. In data transmission duration, hello packets are transmitted periodically to maintain the transmission route. When the data transmission periods are completed both in node $\mathrm{A}$ and in node $\mathrm{B}$, they send ACK in maximum power as notification to neighbor nodes after SIFS period. There is a distributed coordination function (DCF) inner frame space (DIFS) duration after ACK at the end of the transmission period.

In unidirectional route transmission (Figure 6), if node A wants to send data to node B and node B has a data transmission task to node $\mathrm{C}$, node $\mathrm{A}$ will send RTS to start the transmission. After SIFS duration, node B sends CTS with its location information in its maximum power. Node $\mathrm{C}$ can also receive the CTS from node $\mathrm{B}$ because it is in the STR of node $\mathrm{B}$. When the CTS from node $\mathrm{C}$ is successfully received by node $\mathrm{B}$, the data transmission starts after SIFS duration. The data transmission from node $\mathrm{A}$ to node $\mathrm{B}$ and that form node $\mathrm{B}$ to node $\mathrm{C}$ start simultaneously. When both of the transmissions are finished, node $\mathrm{B}$ and node $\mathrm{C}$ send ACK packets after SIFS period, and there is a DIFS period after the transmission of ACK. 


\section{Analysis and Simulations}

4.1. Energy Consumption Analysis. In this paper, the presented model is described as a connectivity graph $G(V, E)$, where $V$ is a finite set of nodes and $(i, j) \in E$ is a wireless transmission link between node $i$ and node $j$. The node state information is represented as

$$
\Psi i(t)=f\left(\left(x(i, t), y(i, t), v(i, t), R_{i}\right)\right),
$$

where $f(x)$ is the function of node state, $x(i, t)$ and $y(i, t)$ represent the position of node $i$ at time $t$ in Cartesian coordinate system, $v(i, t)$ is the node's speed at time $t$, and $R_{i}$ is the transmission range, which includes STR and DTR in this paper.

In the initialization period, the sender node broadcasts discovery message with its power being $P_{\mathrm{DM}}$. Thus, the power consumed in the initialization period can be described as

$$
P_{\mathrm{INT}}=\sum_{i \in N_{S}} P_{\mathrm{DM}}\left(\Psi i\left(t_{S}+t_{\mathrm{DM}}\right)\right)
$$

where $N_{S}$ is the set of all sender nodes in the model, $t_{S}$ is the time stamp, and $t_{\mathrm{DM}}$ is the time spent on broadcasting discovery message.

The RTS, CTS, RRP, and ACK packets are all defined as control packets in this paper. The energy consumed in control packets can be represented as

$$
\begin{aligned}
P_{\mathrm{CTRL}}= & \sum_{i \in N_{S}} P_{\mathrm{RTS}}\left(\Psi i\left(t_{\mathrm{RTS}}\right)\right)+\sum_{j \in N_{D}} P_{\mathrm{CTS}}\left(\Psi j\left(t_{\mathrm{CTS}}\right)\right) \\
& +\sum_{j \in N_{D}} P_{\mathrm{RRP}}\left(\Psi j\left(t_{\mathrm{RRP}}\right)\right) \\
& +\sum_{j \in N_{D}} P_{\mathrm{ACK}}\left(\Psi j\left(t_{\mathrm{ACK}}\right)\right),
\end{aligned}
$$

where $N_{D}$ is the set of all destination nodes and $t_{\mathrm{RTS}}, t_{\mathrm{CTS}}$, $t_{\mathrm{RRP}}$, and $t_{\mathrm{ACK}}$ represent the time spent on RTS, CTS, RRP, and ACK, respectively. Because the control packets are all in STR, the transmission power needs to be adjusted to $P_{\max }$.

$P_{\text {DATA-P }}$ is the power spent on data transmission period in the presented model, which contains two parts: data transmission and hello packets transmission, and the energy consumption is $P_{\mathrm{DATA}}$ and $P_{\mathrm{HEL}}$, respectively. $N_{P}$ is the set of nodes which have packets to transmit and $t_{\text {DATA }}$ and $t_{\mathrm{HEL}}$ are the time spent on data transmission and hello packets transmission. So, the total power spending on data transmission period is shown:

$$
\begin{aligned}
P_{\mathrm{DATA}-\mathrm{P}}= & P_{\mathrm{DATA}}+P_{\mathrm{HEL}} \\
= & \sum_{i \in N_{P}} P_{\mathrm{DATA}}\left(\Psi i\left(t_{\mathrm{DATA}}\right)\right) \\
& +\sum_{i \in N_{P}} P_{\mathrm{HEL}}\left(\Psi i\left(t_{\mathrm{HEL}}\right)\right) .
\end{aligned}
$$

As mentioned above, the energy consumption in the whole transmission can be represented as

$$
P_{\mathrm{TRANS}}=P_{\mathrm{INT}}+P_{\mathrm{CTRL}}+P_{\mathrm{DATA}-\mathrm{P}} .
$$

4.2. Performance Analysis on Transmission. The throughput of the present model is defined as

$$
\mathrm{Th}=\frac{m_{d} \sum_{j \in N_{D}} N_{j \mathrm{DP}}}{t_{\mathrm{op}}} .
$$

Th is the model throughput, $m_{d}$ (bit) is the length of every data packet, $N_{j \mathrm{DP}}$ is the number of received packets in node $j$, and $t_{\text {op }}$ is the network operation time.

PDR represented packets delivery rate. PDR shows the percentage of successful transmission in presented model and is a significant performance to evaluate the model. PDR can be calculated as

$$
\operatorname{PDR}(\%)=\frac{\sum_{j \in N_{D}} N_{j \mathrm{DP}}}{\sum_{i \in N_{S}} N_{i \mathrm{SP}}} \times 100 \%,
$$

where $N_{j \mathrm{DP}}$ is the number of packets received by destination node $j$ and $N_{i S P}$ is the number of packets sent by sender node $i$.

End-to-end delay in the presented model is represented as

$$
t_{\text {EEdelay }}=\frac{\sum_{i \in N_{\mathrm{SCS}}} t_{i \text { delay }}}{n} .
$$

It denotes the average delay time of every successfully transmitted packet, $N_{\mathrm{SCS}}$ is the set of successful transmission nodes, $t_{\text {idelay }}$ is the end-to-end delay of node $i$, and $n$ is the number of successful transmission packets.

$\mathrm{CPR}$ is short for control packets rate, and it can be calculated as follows:

$$
\mathrm{CPR}(\%)=\frac{m_{c} \sum_{i \in N_{\mathrm{SCS}}} N_{i \mathrm{CP}}}{m_{d} \sum_{i \in N_{\mathrm{SCS}}} N_{i \mathrm{DP}}} \times 100 \%,
$$

where $N_{i \text { CP }}$ is the number of control packets sent by node $i$ and $N_{i \mathrm{DP}}$ is the number of data packets successfully sent by node $i, m_{d}$ (bit) is the length of every data packet, and $m_{c}$ (bit) is the length of every control packet. A low CPR will lead to low energy consumption when other parameters in the model remain unchanged.

In the MWSN, we use fair index (FI) to evaluate the fairness of nodes accessing the channel, and it indicates the fairness of nodes accessing the networks. FI is presented as

$$
\mathrm{FI}=\frac{\left(\sum_{i} \mathrm{Th}_{i}\right)^{2}}{n \sum_{i} \mathrm{Th}_{i}^{2}},
$$

where $n$ is the number of contending nodes and $\mathrm{Th}_{i}$ is the throughput of node $i$. According to Cauchy-Schwarz inequality, $\mathrm{FI} \leq 1$, and only when $\mathrm{Th}_{i}=\mathrm{Th}_{j}(i \neq j), \mathrm{FI}=1$. Therefore, the fairness of the protocol will become better as FI approaches 1.

4.3. Simulations. In this section, we assume the simulation environment of propagation model as follows:

(1) All transmission happens in Rayleigh fading channels with line-of-sight transmission. 


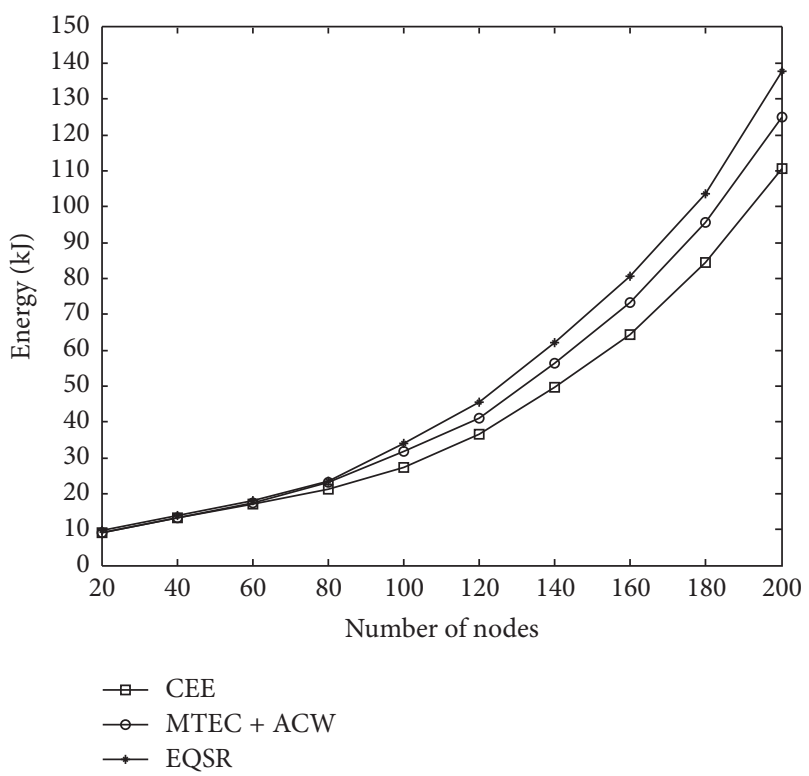

FIGURE 7: Total network energy consumption.

(2) All sensor nodes in the model are mobile and equipped with two interfaces.

(3) The deployment surface of the model is flat.

(4) The model system is homogenous, and the nodes are of the same type and capabilities.

(5) The length of every data packet is 1024 bits, and the lengths of RTS, CTS, and ACK are all 64 bits.

(6) The specific durations of transmission are as follows: $28 \mu \mathrm{s}$ for SIFS, $128 \mu \mathrm{s}$ for DIFS, $50 \mu$ s for slot duration, and $20 \mu$ s for hello packet. The period of hello packets is $340 \mu \mathrm{s}$.

The proposed model has been evaluated by NS-3.26 simulator. The transmission scenario in the simulation is built as the IoT, and it has a deployment area of $300 \times 300$ square meters. The initial energy of every node is 1000 Joules, and the largest queue length is 150 packets in every node. The transmission bandwidth is $1 \mathrm{Mbps}$ in the simulation, and the simulation time is 500 seconds. We compare our model CEE against MTEC + ACW and EQSR in this section.

Figure 7 shows the energy consumption of different transmission models. It is clear to see the energy consumption increases with the increasing number of nodes. More nodes lead to more transmission routes in the system, which cause higher energy consumption. When the number of nodes is small (less than 100), the energy consumption of the three models is almost equal. However, when the nodes are in a large scale, CEE performs better, because CEE reduces the control packets rate $(\mathrm{CPR}$, shown in Figure 11) of the model and has higher packets delivery rate (PDR, shown in Figure 9) compared to others, which can reduce energy consumption of the model. MTEC + ACW consumes slightly more energy due to contending channel and EQSR spends a little more energy due to route choosing, so they consume more energy than CEE, especially when the number of nodes

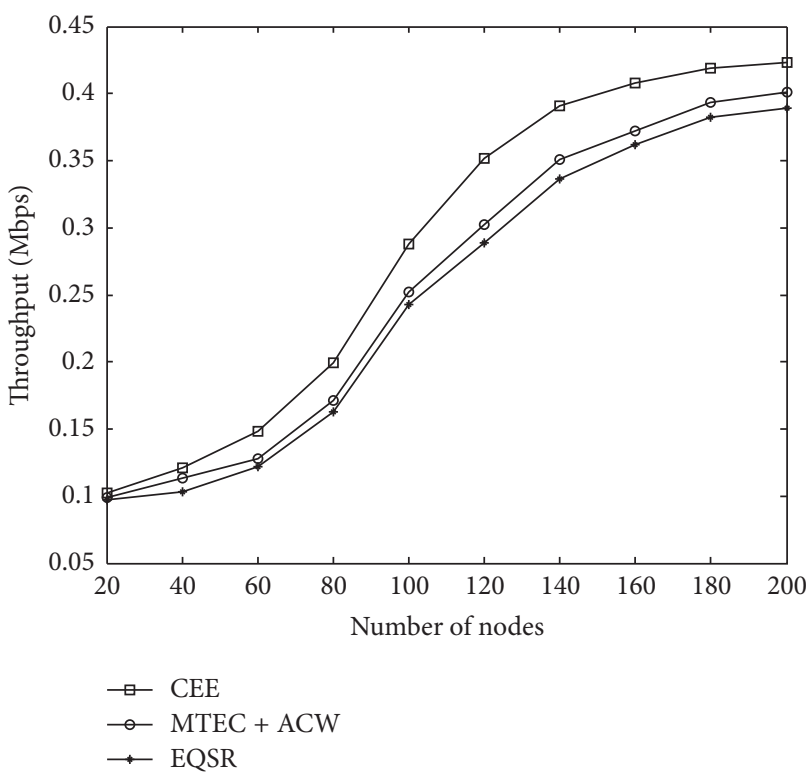

FIGURE 8: Network total throughput.

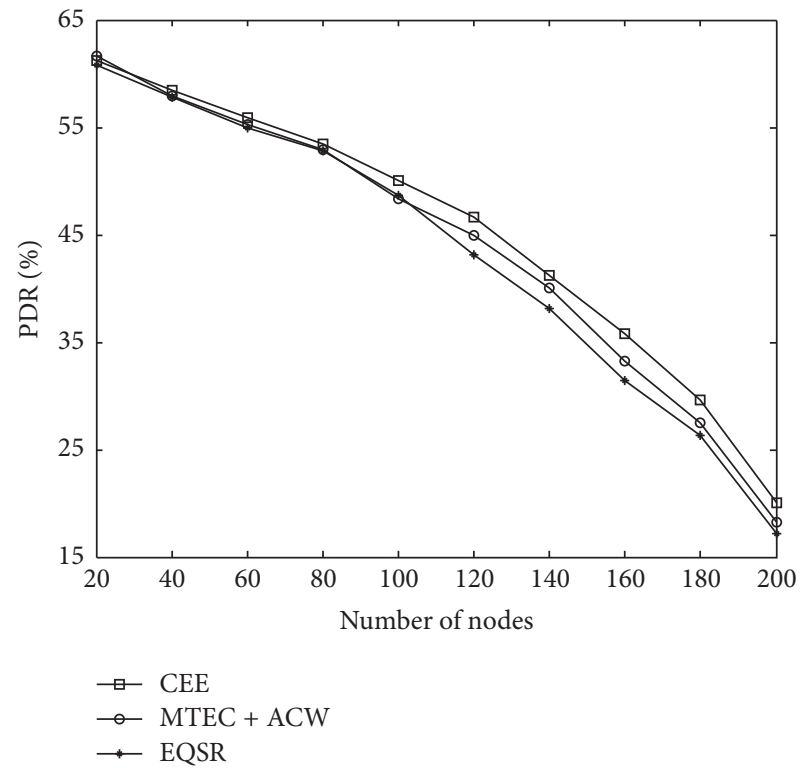

Figure 9: Packet delivery ratio (\%).

is greater than 120 . It is clear to see that, in a transmission model with 200 nodes, CEE has a significant advantage on the energy consumption, and it can save about $25 \%$ energy compared to EQSR and 12\% energy compared to MTEC + ACW, respectively. We can predict that CEE will show its more significant advantage when the number of nodes is more than 200. It is very useful to practical applications, since the development direction of MWSN is to large scale, such as IoT.

Figures 8 and 9 show the throughput and PDR of the three transmission models. The proposed transmission model has a better performance compared to others on throughput and PDR. It is clear to find that CEE is obviously higher on 


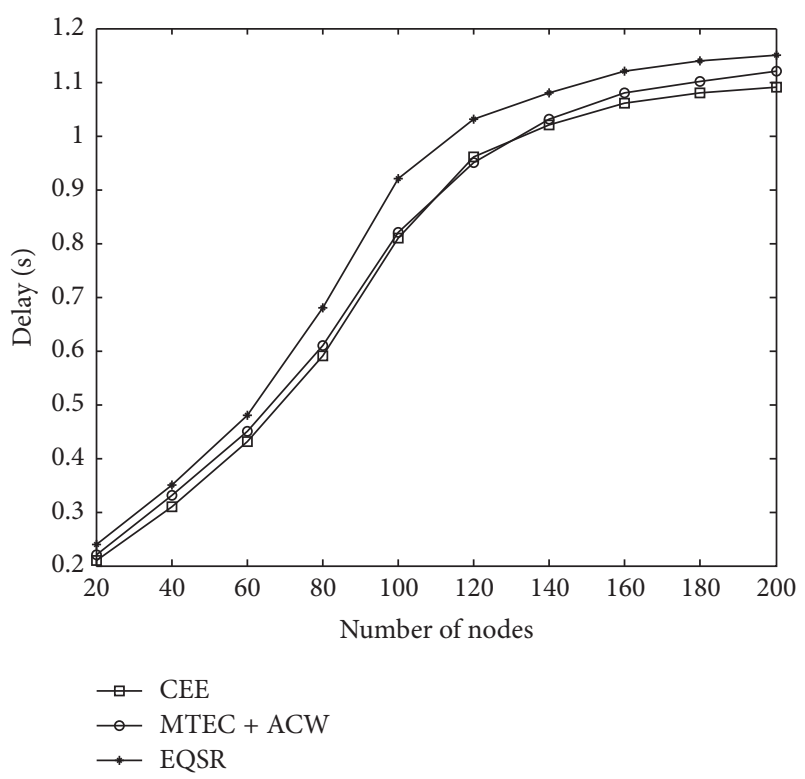

FIGURE 10: End-to-end delay of network.

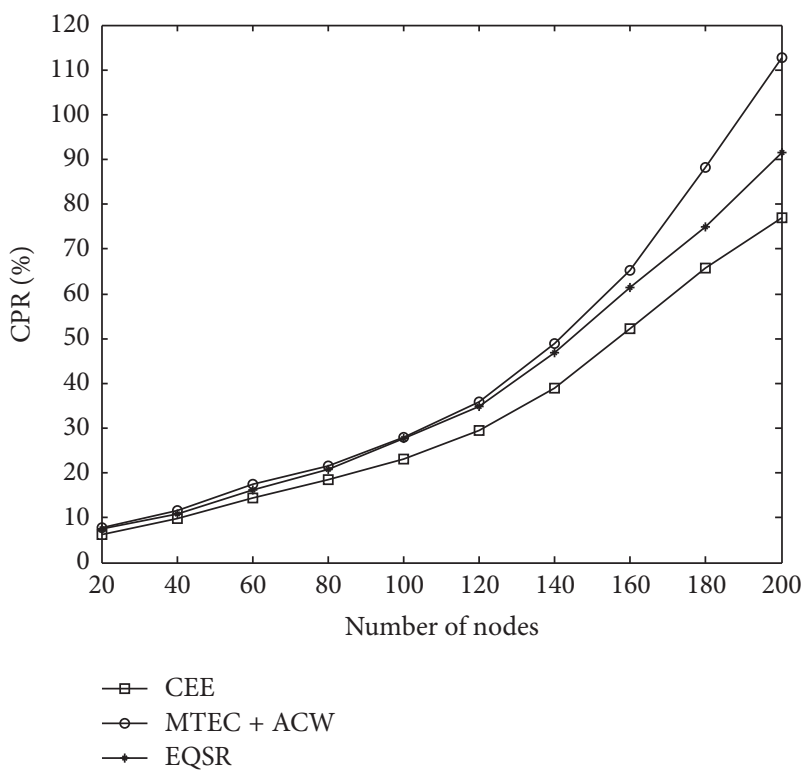

Figure 11: Control packets ratio (\%).

the PDR compared to others when the number of nodes is more than 100. Under the effect of PDR, the throughput of CEE is obviously higher than other models when the number of nodes is from 100 to 140 too. The reason is that CEE utilizes cross-layer model to reduce both control packets overhead and channel occupation. As a result, CEE can provide about $20 \%$ and $30 \%$ more throughput than MTEC + ACW and EQSR, respectively. Higher throughput and PDR can provide faster and more effective quality of service. It is very important to transmit a large amount of data, and it can be useful to big data technology.

End-to-end delay simulation results are shown in Figure 10. The delay increases with the increasing number of

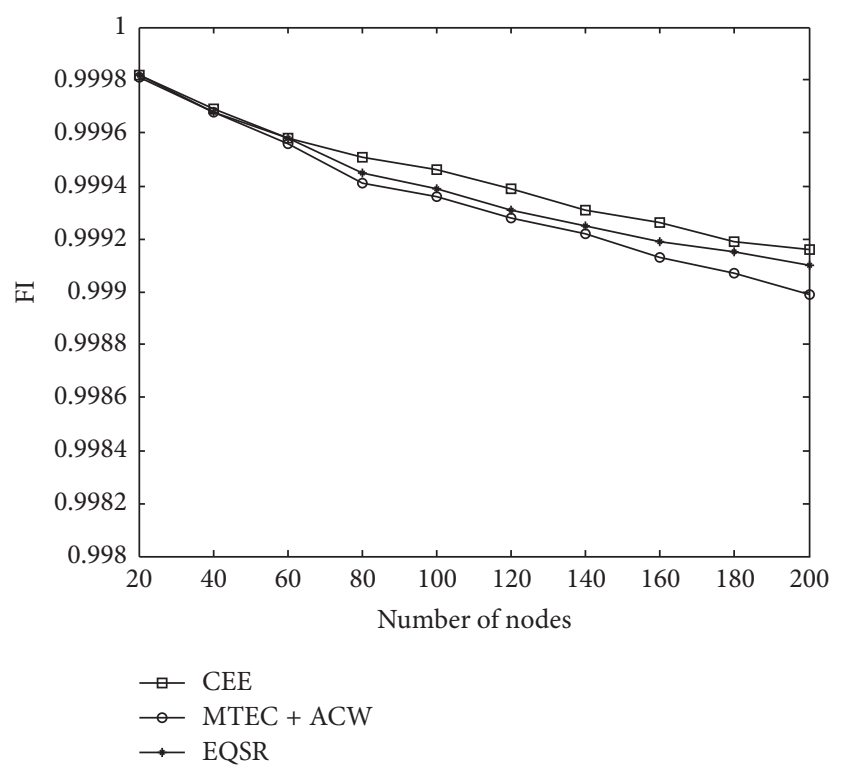

FIGURE 12: FI.

nodes. High CPR and transmission congestion can increase end-to-end delay. CEE has the least CPR of the three models according to Figure 11, which reduces the end-to-end delay of CEE. When the number of nodes is more than 160 , the endto-end delays of the three transmission models all become flat and almost equal. Since the congestion is the main reason for the delay in large scale of nodes. CEE can save nearly 0.1 seconds comparing to the other two models, which improve the delay of about $10 \%$ in large scale networks. Figure 11 shows the CPR of the three models, and the CPR of transmission model depends on its control packets overhead. Low delay of the networks is necessary to MWSN, especially when it is used in disaster relief and some other activities which are highly real-time.

The FI of the three protocols are shown in Figure 12, and they are all greater than 0.9988 . When the number of nodes in networks is small (less than 60), the FI of them are almost equal to each other. With the increasing number of nodes in networks, CEE shows its advantages. The transmission scheme can improve the accessing fair index by relay node initializing transmission. CEE has an obvious advantage compared to others when there are 200 nodes in networks.

In conclusion, CEE not only saves the energy efficiently but also improves the transmission performance of the networks. Based on the performance of proposed transmission model, CEE can be practically applied in the IoT. Most of the user nodes in the IoT are passive nodes and small, and it is important to save the energy of them. However, the networks like IoT also need an outstanding transmission performance. As a result, CEE can solve both of the problems at the same time.

\section{Conclusion}

In this paper, an energy efficiency cross-layer transmission model for mobile wireless sensor network CEE is proposed. 
The transmission model integrates three layers in network operation. The first is network layer (nodes' location information), the second is data link layer (MAC layer protocol), and the third is PHY layer (interfaces of nodes).

According to the theoretical analysis and numerical simulations, the proposed transmission model not only has high energy efficiency but also has a better transmission performance on throughput, PDR, delay, and CPR. Moreover, CEE solves energy consumption problem for mobile wireless sensor networks without negative effect on other transmission performances. In the future, we will study on the practical applications of the proposed cross-layer transmission model on MWSN such as the large scale IoT and other application scenarios.

\section{Conflicts of Interest}

The authors declare that there are no conflicts of interest regarding the publication of this paper.

\section{Acknowledgments}

This work was supported in part by the National Natural Science Foundation of China (nos. 61271416, 61301093, and 61601372).

\section{References}

[1] M. Arifuzzaman, M. Matsumoto, and T. Sato, "An intelligent hybrid MAC with traffic-differentiation-based QoS for wireless sensor networks," IEEE Sensors Journal, vol. 13, no. 6, pp. 23912399, 2013.

[2] S. Ping, A. Aijaz, O. Holland, and A.-H. Aghvami, "SACRP: a spectrum aggregation-based cooperative routing protocol for cognitive radio Ad-Hoc networks," IEEE Transactions on Communications, vol. 63, no. 6, pp. 2015-2030, 2015.

[3] J. Wang, D. Li, G. Xing, and H. Du, "Cross-layer sleep scheduling design in service-oriented wireless sensor networks," IEEE Transactions on Mobile Computing, vol. 9, no. 11, pp. 1622-1633, 2010.

[4] C.-C. Weng, C.-W. Chen, P.-Y. Chen, and K.-C. Chang, "Design of an energy-efficient cross-layer protocol for mobile ad hoc networks," IET Communications, vol. 7, no. 3, pp. 217-228, 2013.

[5] L. Karim and N. Nasser, "Reliable location-aware routing protocol for mobile wireless sensor network," IET Communications, vol. 6, no. 14, pp. 2149-2158, 2012.

[6] L. Zhang and Y. Zhang, "Energy-efficient cross-layer protocol of channel-aware geographic-informed forwarding in wireless sensor networks," IEEE Transactions on Vehicular Technology, vol. 58, no. 6, pp. 3041-3052, 2009.

[7] X. Ji, Y. He, J. Wang, W. Dong, X. Wu, and Y. Liu, "Walking down the STAIRS: efficient collision resolution for wireless sensor networks," in Proceedings of the IEEE Conference on Computer Communications (INFOCOM '14), pp. 961-969, IEEE, Toronto, Canada, April 2014.

[8] J. Ben-Othman and B. Yahya, "Energy efficient and QoS based routing protocol for wireless sensor networks," Journal of Parallel and Distributed Computing, vol. 70, no. 8, pp. 849-857, 2010.
[9] "IEEE Standard for Information Technology- Telecommunications and Information Exchange Between Systems-Local and Metropolitan Area Networks-Specific Requirements-Part 11: Wireless LAN Medium Access Control (MAC) and Physical Layer (PHY) Specifications," IEEE Std 802.11, 2007.

[10] X. Yang and L. Wang, "A multichannel transmitting and assistant nodes MAC protocol for mobile ad hoc networks," in Proceedings of the IEEE Global Communication Conference (GLOBECOM '15), San Diego, Calif, USA, December 2015.

[11] V. Nguyen, O. T. T. Kim, T. N. Dang, and C. S. Hong, "Improving time slot acquisition through RSU's coordination for TDMAbased MAC protocol in VANETs," in Proceedings of the 30th International Conference on Information Networking (ICOIN '16), pp. 406-411, IEEE, Kota Kinabalu, Malaysia, January 2016.

[12] S. H. R. Bukhari, M. H. Rehmani, and S. Siraj, "A survey of channel bonding for wireless networks and guidelines of channel bonding for futuristic cognitive radio sensor networks," IEEE Communications Surveys and Tutorials, vol. 18, no. 2, pp. 924-948, 2016

[13] S. Debroy, S. De, and M. Chatterjee, "Contention based multichannel MAC protocol for distributed cognitive radio networks," IEEE Transactions on Mobile Computing, vol. 13, no. 12, pp. 2749-2762, 2014.

[14] H. A. Omar, W. Zhuang, and L. Li, "VeMAC: A TDMAbased MAC protocol for reliable broadcast in VANETs," IEEE Transactions on Mobile Computing, vol. 12, no. 9, pp. 1724-1736, 2013.

[15] W. Li, M.-L. Ku, Y. Chen, and K. J. Ray Liu, "On outage probability for two-way relay networks with stochastic energy harvesting," IEEE Transactions on Communications, vol. 64, no. 5, pp. 1901-1915, 2016.

[16] Z.-T. Li, Q. Chen, G.-M. Zhu, Y.-J. Choi, and H. Sekiya, "A low latency, energy efficient MAC protocol for wireless sensor networks," International Journal of Distributed Sensor Networks, vol. 2015, Article ID 946587, 9 pages, 2015.

[17] Y. Lee, E.-C. Park, and C.-H. Choi, "Signaling-free maxmin airtime fairness in IEEE 802.11 ad hoc networks," Mobile Information Systems, vol. 2016, Article ID 9586893, 19 pages, 2016.

[18] J.-H. Noh and S.-J. Oh, "Beamforming in a multi-user cognitive radio system with partial channel state information," IEEE Transactions on Wireless Communications, vol. 12, no. 2, pp. 616625, 2013.

[19] R. S. Chib, Y. Singh, S. Singh, and R. Gupta, "Performance \& Evaluation of propagation models for sub-urban areas," International Journal of Advanced Research in Electrical, Electronics and Instrumentation Engineering, vol. 4, no. 2, pp. 607-614, 2015.

[20] W. Guibène and D. Slock, "Cooperative spectrum sensing and localization in cognitive radio systems using compressed sensing," Journal of Sensors, vol. 2013, Article ID 606413, 9 pages, 2013. 

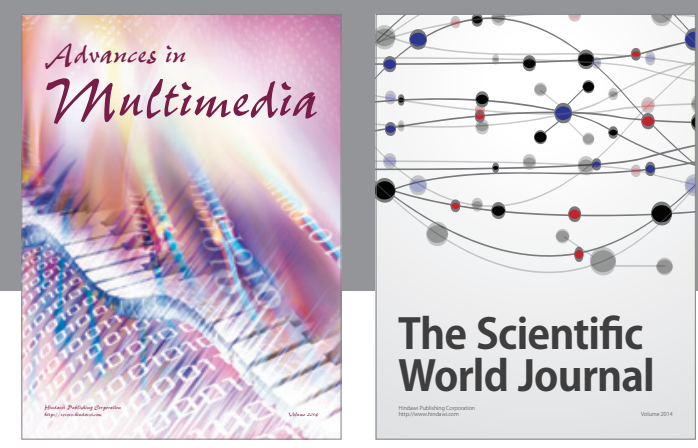

The Scientific World Journal
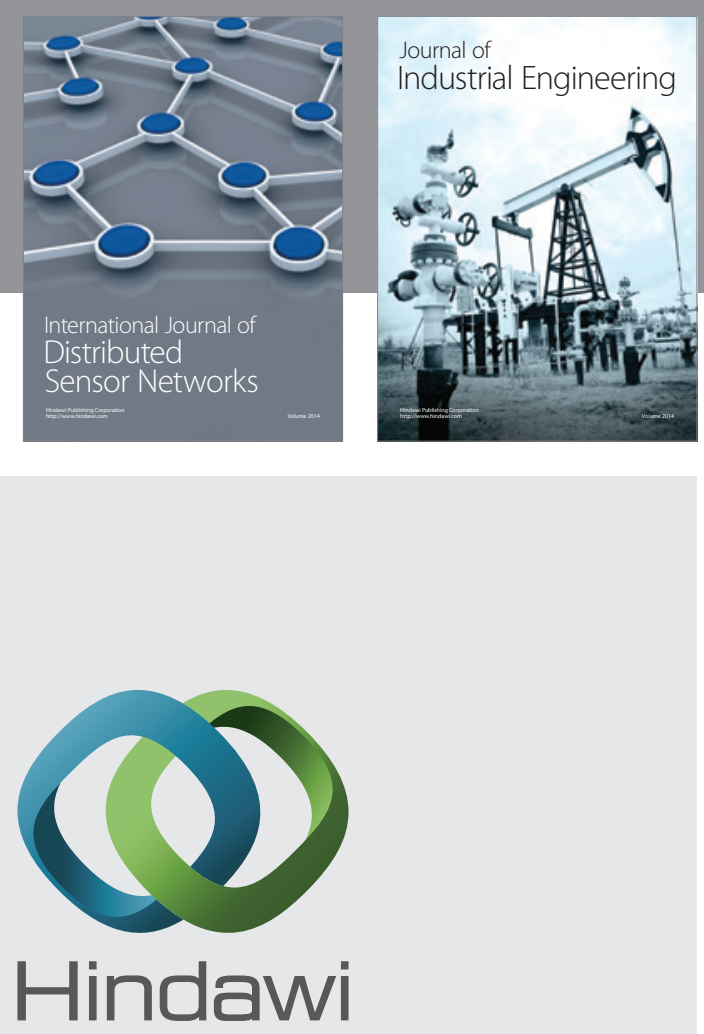

Submit your manuscripts at

https://www.hindawi.com

\section{Computer Networks} and Communications
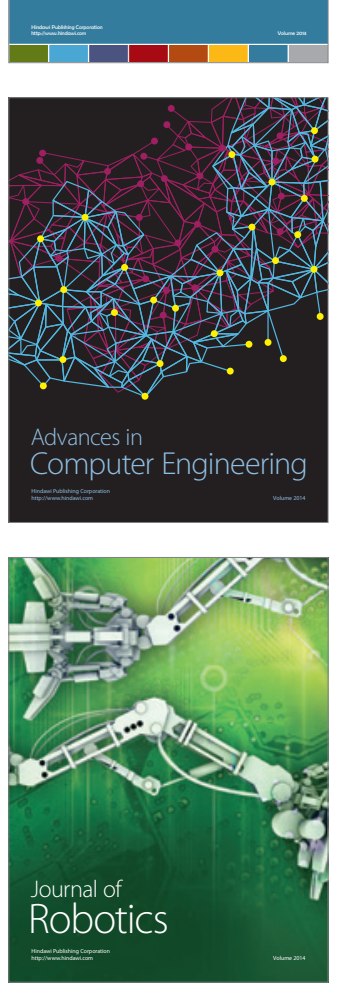
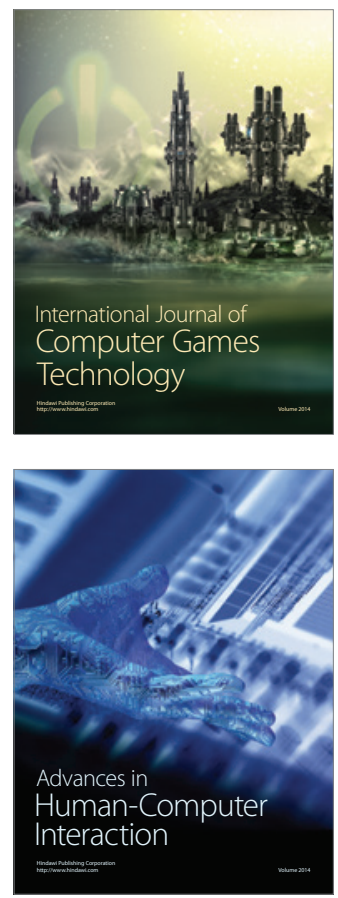
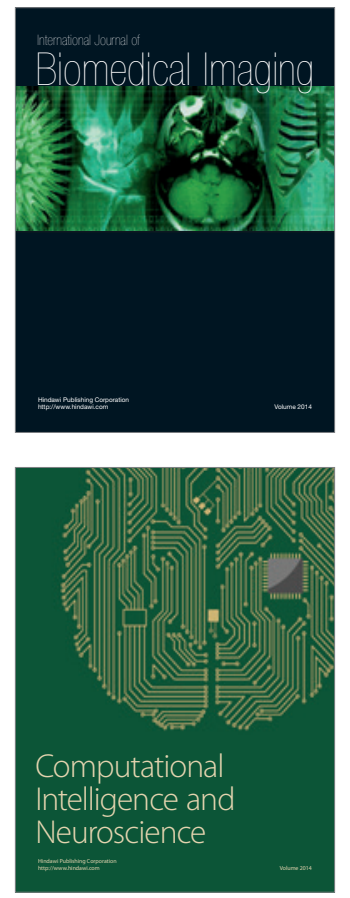
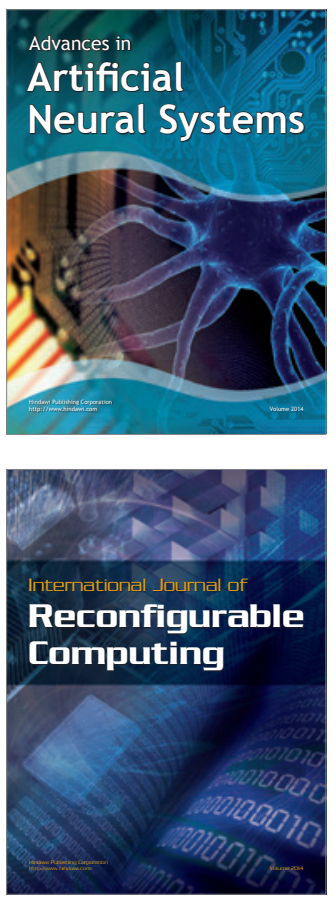
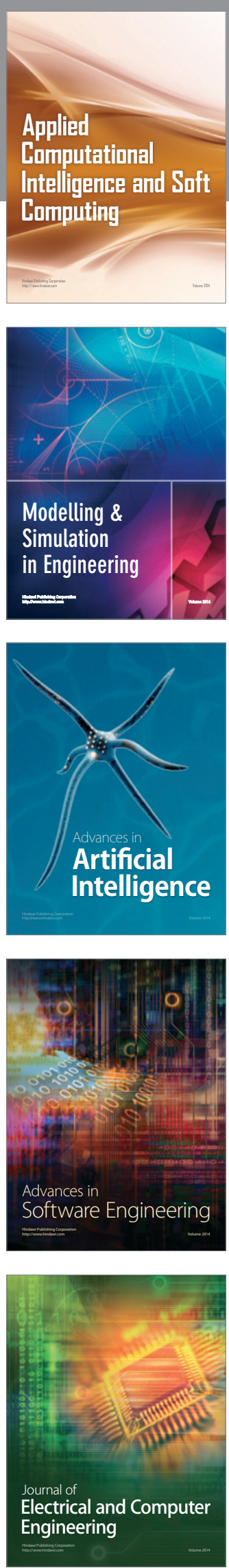